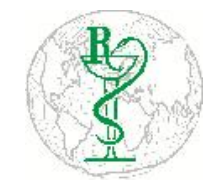

INDO GLOBAL JOURNAL OF

PHARMACEUTICAL SCIENCES

ISSN 2249- 1023

\title{
Synthesis, Alpha Amylase Inhibition, Antimicrobial, Antioxidant and Molecular Docking Studies of $\boldsymbol{p}$-Coumaric Acid Derivatives
}

\author{
Shashi Sharma, Kalyan Mondal, Anurag Khatkar, Saloni Kakkar* \\ Department of Pharmaceutical Sciences, M D University, Rohtak (Haryana), India
}

\begin{abstract}
Address for
Correspondance

Saloni Kakkar,

$\underline{\text { salonikakkar2007 }}$

@ gmail.com

Received:

17.02.2017

Accepted:

30.04.2017

ABSTRACT: A series of $p$-coumaric acid derivatives were designed to yield compounds having antidiabetic, antimicrobial and antioxidant activity. Here, we synthesized derivatives, docked them and evaluated all the synthesized products by biological methods. Results of this study revealed that amongst all the compounds, compound 1 displayed activity against inhibition of pancreatic alpha amylase, antimicrobial, antioxidant and showed maximum binding energy. For docking studies AutoDock4.2 was used, for inhibition test of alpha amylase activity, calorimetric method was adopted. Antimicrobial activity was evaluated by tube dilution method and antioxidant activity was done by DPPH free radical assay. () 2017 iGlobal Research and Publishing Foundation. All rights reserved.
\end{abstract}

Keywords p-Coumaric Acid; Alpha Amylase; Diabetes; Binding Energy; Molecular Docking.

\section{INTRODUCTION}

Type 2 diabetes (T2DM) is most common form of diabetes mellitus and characterized by hyperlipidemia, hyperglycemia, insulin resistance, high blood glucose level and glycosuria [15]. T2DM was formerly called adult-onset or non-insulindependent, resulting from ineffective insulin used by body [1]. $\alpha-1,4-$ Glycosidic bonds are broken down by $\alpha$-amylase which is a glycoside hydrolase enzyme. Hydrolyses of disaccharides to monosaccharide can be done by $\quad \alpha$-glucosidase and absorbed into the blood stream [2]. $\alpha$-amylase and $\alpha$ glucosidase acts as key enzyme for the absorption and digestion of carbohydrates and for the modulation of the pathologic postprandial hyperglycemia, the enzymes have been identified as important therapeutic targets for T2DM suffering patients [1].

Plants are the rich sources of hypoglycemic, hypolipidemic compounds, antioxidants such as phenolic acids and related polyphenols. [3]. $P$-coumaric acid is the most common hydroxycinnamic acid widely distributed in foods, fruit juices and vegetables [4]. $P$-coumaric acid exhibits interesting pharmacological activities including antioxidant [5], antimicrobial [6], antidiabetic [7], anticancer [8], antiinflammatory [9], antihypertensive [10], antiulcer [11] and antimelanogenic [12].

The aim of the present research is to synthesize various $p$ coumaric acid derivatives and evaluate them for antidiabetic, antimicrobial and antioxidant potential.

\section{MATERIALS AND METHODS}

To prove the feasibility of the synthesis, experiments were performed using various solvents like thionyl chloride, toluene, ether and methanol etc. All reagents and solvents used in study were of analytical grade and procured locally. Melting points were determined in open capillary tubes on a Sonar melting point apparatus and are uncorrected. Reaction progress was monitored by thin layer chromatography on silica gel coated glass plate and the purity of the compounds was ascertained by single spot on TLC sheet. $\mathrm{H}^{1}$ nuclear magnetic resonance $\left(\mathrm{H}^{1} \mathrm{NMR}\right)$ spectra were recorded on 
Indo Global Journal of Pharmaceutical Sciences, 2017; 7(2): 126-133

Agilent NMR $4300 \mathrm{MHz}$ spectrometer using DMSO-d6 solvent and are expressed in parts per million $(\delta$, ppm). Infrared (IR) spectra were recorded on a Shimadzu FT-IR spectrophotometer.

General procedure for synthesis of (E)-3-(4-hydroxyphenyl) acryloyl chloride.

Thionyl chloride $(0.15 \mathrm{~mol})$ was added gently to $p$-coumaric acid $(0.125 \mathrm{~mol})$ in a round bottom flask. Then after addition of thionyl chloride, the mixture was stirred for 4 to 5 hours and heated to $80{ }^{\circ} \mathrm{C}$ for $30-40 \mathrm{~min}$ in water bath. The end point of the reaction was detected by using thin layer chromatography. After completion of the reaction, solid mass was filtered and washed with toluene to obtain $(E)-3-(4-$ hydroxyphenyl) acryloylchloride.

General procedure for synthesis of (E)- quinolin-8-yl 3-(4hydroxyphenyl) acrylate

The solution of 8-hydroxy quinolin $(0.005 \mathrm{~mol})$ in ether $(7 \mathrm{~mL})$ was added to a solution of (E)-3-(4-hydroxyphenyl) acryloylchloride $(0.15 \mathrm{~mol})$ in ether $(7 \mathrm{~mL})$. Then the reaction mixture was heated to $80-82^{\circ} \mathrm{C}$ for $60 \mathrm{~min}$ in water bath. The reaction monitoring was done using TLC. The mixture was cooled at $37^{\circ} \mathrm{C}$ and evaporation of solvent yielded the crude ester which was purified by recrystallization with alcohol.
General procedure for synthesis of amides.

The solution of corresponding amine $(0.005 \mathrm{~mol})$ in ether $(7$ $\mathrm{mL})$ was added dropwise to a solution of (E)-3-(4hydroxyphenyl) acryloyl chloride $(0.15 \mathrm{~mol})$ in ether $(7 \mathrm{~mL})$ and maintained temperature at $0-10{ }^{\circ} \mathrm{C}$ (Scheme 1). The reaction mixture was stirred for $50-60 \mathrm{~min}$ and the precipitated amide was separated out. The crude amide, 2-14 (Table 1) was recrystallized with alcohol.<smiles>O=C(O)/C=C/c1ccc(O)cc1</smiles><smiles>O=C(Cl)/C=C/c1ccc(O)cc1</smiles><smiles>Cc1cnc2c(O)cccc2c1C</smiles>

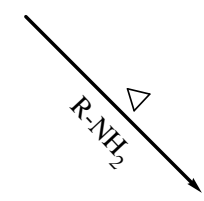<smiles>O=C(/C=C/c1ccc(O)cc1)Oc1cccc2cccnc12</smiles>

1<smiles>[R]OC(=O)/C=C/c1ccc(O)cc1</smiles>

2-14
Scheme 1: Synthesis of $p$-coumaric acid derivatives

Table 1: Substituent used for targeted compounds

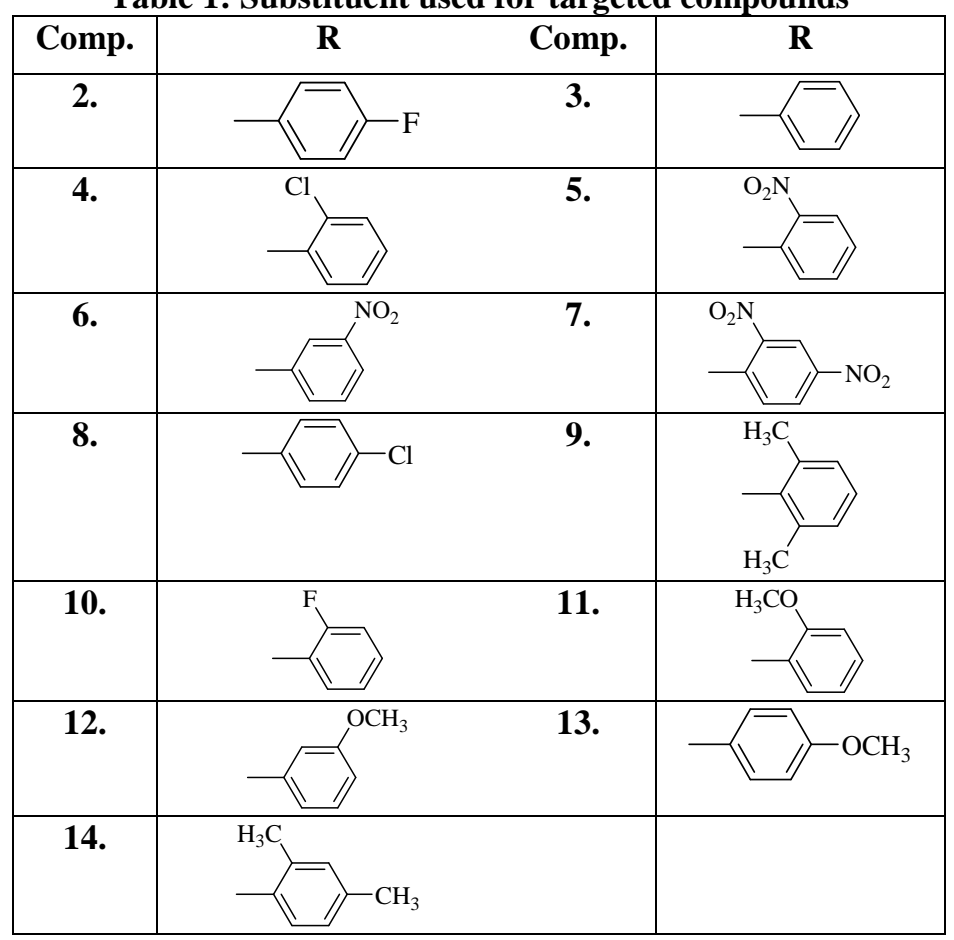


Indo Global Journal of Pharmaceutical Sciences, 2017; 7(2): 126-133

Table 2: Physicochemical properties of synthesized $p$-coumaric acid derivatives (1-14)

\begin{tabular}{|c|c|c|c|c|c|}
\hline Comp. & Mol. Formula & M. Wt. & m.p. $\left({ }^{\mathbf{0}} \mathbf{C}\right)$ & $\mathbf{R}_{\mathbf{f}}$ Value & \% Yield \\
\hline 1. & $\mathrm{C}_{18} \mathrm{H}_{13} \mathrm{NO}_{2}$ & 291.13 & $212-215$ & 0.42 & 78.40 \\
2. & $\mathrm{C}_{15} \mathrm{H}_{12} \mathrm{FNO}_{2}$ & 257.26 & $217-219$ & 0.59 & 68.96 \\
3. & $\mathrm{C}_{16} \mathrm{H}_{15} \mathrm{NO}_{2}$ & 253.3 & $219-223$ & 0.45 & 62.06 \\
4. & $\mathrm{C}_{16} \mathrm{H}_{14} \mathrm{ClNO}_{2}$ & 287.74 & $228-230$ & 0.80 & 97.77 \\
5. & $\mathrm{C}_{16} \mathrm{H}_{14} \mathrm{~N}_{2} \mathrm{O}_{4}$ & 298.29 & $232-234$ & 0.81 & 53.79 \\
6. & $\mathrm{C}_{15} \mathrm{H}_{12} \mathrm{~N}_{2} \mathrm{O}_{4}$ & 284.27 & $237-239$ & 0.21 & 59.09 \\
7. & $\mathrm{C}_{15} \mathrm{H}_{11} \mathrm{~N}_{3} \mathrm{O}_{6}$ & 329.26 & $243-245$ & 0.58 & 81.37 \\
8. & $\mathrm{C}_{15} \mathrm{H}_{12} \mathrm{ClNO}_{2}$ & 273.71 & $250-252$ & 0.44 & 70.34 \\
9. & $\mathrm{C}_{17} \mathrm{H}_{17} \mathrm{NO}_{2}$ & 267.32 & $257-258$ & 0.52 & 50.34 \\
10. & $\mathrm{C}_{15} \mathrm{H}_{12} \mathrm{FNO}_{2}$ & 257.26 & $261-263$ & 0.71 & 56.81 \\
1. & $\mathrm{C}_{16} \mathrm{H}_{15} \mathrm{NO}_{3}$ & 269.3 & $267-269$ & 0.36 & 75.55 \\
12. & $\mathrm{C}_{16} \mathrm{H}_{15} \mathrm{NO}_{3}$ & 269.3 & $271-273$ & 0.50 & 79.44 \\
13. & $\mathrm{C}_{16} \mathrm{H}_{15} \mathrm{NO}_{3}$ & 269.3 & $275-278$ & 0.20 & 69.65 \\
14. & $\mathrm{C}_{17} \mathrm{H}_{17} \mathrm{NO}_{2}$ & 267.32 & $283-285$ & 0.46 & 57.95 \\
\hline
\end{tabular}

*TLC mobile phase:-Chloroform:Benzene (3:7)

\section{RESULTS AND DISCUSSION}

Physicochemical properties of the synthesized derivatives of $\mathrm{p}$ coumaric acid was tabulated in Table 2.

Antidiabetic activity: The inhibition test for alpha amylase was done by calorimetric method [13]. Briefly, the assay mixture contained phosphate buffer $\mathrm{pH} 6.9$ [14]. $1 \mathrm{ml}$ of each synthesized compounds and $1 \mathrm{ml}$ enzyme solution were mixed in a test tube and incubated at $25{ }^{\circ} \mathrm{C}$ for $10 \mathrm{~min}$. To $1 \mathrm{ml}$ of this mixture was added $1 \mathrm{ml}$ of starch solution and the test tube was incubated at 25 ${ }^{0} \mathrm{C}$ for $10 \mathrm{~min}$. Then, $1 \mathrm{ml}$ of colour reagent was added and the closed test tube was placed into water bath at $85{ }^{\circ} \mathrm{C}$ for $10 \mathrm{~min}$. After $15 \mathrm{~min}$, the reaction mixture was removed from the water bath, cooled and diluted with $9 \mathrm{ml}$ distilled water and the absorbance value was determined at $540 \mathrm{~nm}$ in UV spectrophotometer. Acarbose solution was used as positive control. Compounds concentration providing 50\% inhibition $\left(\mathrm{IC}_{50}\right)$ was calculated from the graph plotted as inhibition percentage against compound concentration [15]. All the synthesized compounds were tested against diastase amylase by using calorimetric method and $\mathrm{IC}_{50}$ are shown in Table 3. Acarbose was used as a standard in the assay with $\mathrm{IC}_{50}$ value of $0.19 \mu \mathrm{M} / \mathrm{ml}$. Compound $1(0.21 \mu \mathrm{M} / \mathrm{ml})$, compound $6(0.24 \mu \mathrm{M} / \mathrm{ml})$ were found to be active on comparison with standard. From the results of $\alpha$ amylase inhibitory activity it was found that compound 1 and 6 had good amylase inhibition activity.

The inhibition percentage of $\alpha$-amylase was calculated using following formula:

$$
I \%=\frac{A_{\text {Control }}-\mathbf{A}_{\text {Sample }}}{A_{\text {Control }}} \times 100
$$

Where,

A $_{\text {control: }}$ Absorbance of the control reaction

A sample: $_{\text {: Absorbance of the test compound }}$

Table 3: $\mathrm{IC}_{50}$ value of synthesized compounds for $\alpha$ amylase activity

\begin{tabular}{|c|c|}
\hline Compound & $\mathbf{I C}_{\mathbf{5 0}}$ value $\boldsymbol{\mu M} \mathbf{M} \mathbf{m}$ \\
\hline 1. & 0.21 \\
\hline 2. & 0.25 \\
\hline 3. & 0.38 \\
\hline 4. & 0.30 \\
\hline 5. & 0.29 \\
\hline 6. & 0.24 \\
\hline 7. & 0.27 \\
\hline 8. & 0.32 \\
\hline 9. & 0.39 \\
\hline 10. & 0.29 \\
\hline 11. & 0.35 \\
\hline 12. & 0.27 \\
\hline 13. & 0.31 \\
\hline 14. & 0.32 \\
\hline Acarbose* & 0.19 \\
\hline * Standard & \\
\hline
\end{tabular}

Antimicrobial assay: The synthesized derivatives was evaluated against Gram-positive bacteria: Staphylococcus aureus MTCC 3160, Bacillus subtilis MTCC 441, Gramnegative bacterium: Escherichia coli MTCC 443 and fungal strains: Candida albicans MTCC 227 and Aspergillus niger MTCC 281 using tube dilution method using ciprofloxacin and fluconazole as standard drugs for antibacterial and antifungal activity, respectively [6]. Dilutions of test and standard compounds were prepared in double-strength nutrient 
Indo Global Journal of Pharmaceutical Sciences, 2017; 7(2): 126-133

broth-I.P. (bacteria) or sabouraud dextrose broth I.P. (fungi). The samples were incubated at $37^{\circ} \mathrm{C}$ for $24 \mathrm{hr}$ (bacteria), at $25^{\circ} \mathrm{C}$ for 7 days (A. niger), and at $37^{\circ} \mathrm{C}$ for $48 \mathrm{hr}$ (C. albicans), and the results were recorded in terms of minimum inhibitory concentration (MIC) [16].

All the synthesized compounds (1-14) were investigated for their in vitro antibacterial activity against Gram-positive bacteria (S.aureus, B.subtilis), Gram-negative bacterium (E.coli) and fungal strains (C.albicans and A.niger) by tube dilution method and the results are reported in Table 4. Compounds 1, 2, 8 and 10 showed potent antibacterial activity against the tested strains. In particular, compounds 1 and 8, which possess 8-hydroxy quinoline and 4-chloro groups respectively, were found to be active (MIC: $0.48 \mu \mathrm{M} / \mathrm{ml}$ and $0.49 \mu \mathrm{M} / \mathrm{ml}$ respectively) against E.coli with MIC comparable to Norfloxacin (MIC: $0.47 \mu \mathrm{M} / \mathrm{ml}$ ). Compound 1,2 and 10 were potent against B. subtilis and S.aureus $(0.49 \mu \mathrm{M} / \mathrm{ml}$, $0.48 \mu \mathrm{M} / \mathrm{ml}$ and $0.48 \mu \mathrm{M} / \mathrm{ml}$ respectively) which possess 8hydroxy quinoline, 4-flouro and 2-flouro respectively, as compared to the standard Norfloxacin (MIC: $0.47 \mu \mathrm{M} / \mathrm{ml}$ ), whereas in an antifungal activity it was observed that compound 1 and 5 (MIC:0.51 $\mu \mathrm{M} / \mathrm{ml}$ ) having 8-hydroxy quinoline and 2-nitro groups respectively, showed maximum activity against C.albicans (MIC:0.50 $\mathrm{M} / \mathrm{ml}$ ). Compound 1 and 6 (MIC: $0.51 \mu \mathrm{M} / \mathrm{ml}$ and $0.54 \mu \mathrm{M} / \mathrm{ml}$ respectively) having 8-hydroxy quinoline and 3-nitro group showed activity maximum against A.niger (MIC:0.50 $\mu \mathrm{M} / \mathrm{ml}$ ). From the overall results of antimicrobial activity it was found that compound 1 had maximum antibacterial as well antifungal activity against E. coli, S. aureus and C. albicans, A. niger respectively.

Antioxidant activity: The radical scavenging effect of the stable 1, 1-diphenyl-2-picrylhydrazyl (DPPH)-free radical method was used to determine antioxidant activity of compounds (1-14). The DPPH assay based on free radical DPPH, with an odd electron gives a maximum absorption at $517 \mathrm{~nm}$ (purple colour). When antioxidants react with DPPH, which is a stable free radical becomes paired off in the presence of a hydrogen donor and is reduced to the DPPH-H and as consequence the absorbance decreased from the DPPH. Radical to the DPPH-H form, results in decolorization (yellow colour) with respect to the number of electrons captured. After incubating at $37^{\circ} \mathrm{C}$ for 30 min samples were read against blank at $517 \mathrm{~nm}$ and $\mathrm{IC}_{50}$ was calculated from the graph plotted as inhibition percentage against compound concentration [17].

$$
I \%=\frac{\text { A Control }- \text { A Sample }}{\text { A Control }} \times 100
$$$$
\text { A Control }
$$

Where

$\mathrm{A}_{\text {control }}$ is the absorbance of the control reaction.

A sample is the absorbance of the test compound.

[18]

Tests were carried out in triplicate using ascorbic acid as a positive control. From the results of antioxidant activity, it was observed that compounds 1,4 and 6 have $\mathrm{IC}_{50}$ value comparable to that of positive control ascorbic acid.

Table 4: Minimum Inhibitory Concentration (MIC: $\mu \mathrm{M} / \mathrm{ml}$ ) of (E)-3-(4-hydroxyphenyl) acrylic acid derivatives against bacterial and fungal Strains

\begin{tabular}{|c|c|c|c|c|c|}
\hline $\begin{array}{c}\text { Comp } \\
\text { d. }\end{array}$ & $\begin{array}{c}\mathbf{M I C}_{\mathbf{5 0}(\mathbf{E}} \\
\mathbf{C})\end{array}$ & $\begin{array}{c}\mathbf{M I C}_{\mathbf{5 0}(} \\
\mathbf{B S})\end{array}$ & $\begin{array}{c}\mathbf{M I C}_{\mathbf{5 0}(\mathrm{S}} \\
\mathrm{A})\end{array}$ & $\begin{array}{c}\mathbf{M I C}_{\mathbf{5 0}(\mathrm{C}} \\
\text { A) }\end{array}$ & $\begin{array}{c}\mathbf{M I C}_{\mathbf{5 0}(\mathbf{A}} \\
\mathbf{N})\end{array}$ \\
\hline $\mathbf{1}$ & $\mathbf{0 . 4 8}$ & 0.49 & $\mathbf{0 . 4 9}$ & $\mathbf{0 . 5 1}$ & $\mathbf{0 . 5 1}$ \\
\hline $\mathbf{2}$ & 0.85 & $\mathbf{0 . 4 8}$ & $\mathbf{0 . 4 8}$ & 0.54 & 0.58 \\
\hline $\mathbf{3}$ & 0.98 & 0.98 & 0.57 & 0.98 & 0.98 \\
\hline $\mathbf{4}$ & 0.86 & 0.86 & 0.83 & 0.86 & 0.86 \\
\hline $\mathbf{5}$ & 0.95 & 0.83 & 0.89 & $\mathbf{0 . 5 1}$ & 0.83 \\
\hline $\mathbf{6}$ & 0.59 & 0.87 & 0.87 & 0.59 & $\mathbf{0 . 5 4}$ \\
\hline $\mathbf{7}$ & 0.75 & 0.57 & 1.51 & 0.87 & 0.75 \\
\hline $\mathbf{8}$ & $\mathbf{0 . 4 9}$ & 1.82 & 0.91 & 0.95 & 0.91 \\
\hline $\mathbf{9}$ & 1.46 & 0.93 & 1.46 & 0.93 & 0.86 \\
\hline $\mathbf{1 0}$ & 0.81 & $\mathbf{0 . 4 8}$ & 0.58 & 0.97 & 0.97 \\
\hline $\mathbf{1 1}$ & 0.92 & 0.92 & 0.92 & 0.56 & 0.55 \\
\hline $\mathbf{1 2}$ & 0.98 & 0.98 & 1.85 & 1.85 & 0.57 \\
\hline $\mathbf{1 3}$ & 1.85 & 0.59 & 1.85 & 0.92 & 0.86 \\
\hline $\mathbf{1 4}$ & 0.93 & 1.87 & 0.93 & 0.57 & 0.57 \\
\hline Std & 0.47 & 0.47 & 0.47 & 0.50 & 0.50 \\
\hline
\end{tabular}

${ }^{a}$ Norfloxacin ${ }^{b}$ Fluconazoles, $\mathrm{EC}=E$. coli, $\mathrm{BS}=$ B. subtilis, $\mathrm{SA}=S$. aureus, $\mathrm{CA}=C$. albicans, $\quad \mathrm{AN}=A$. niger

Table 5: \%age Inhibition and $\mathrm{IC}_{50}$ value of synthesized compounds (1-14)

\begin{tabular}{|c|c|c|c|c|c|}
\hline $\begin{array}{c}\text { Compoun } \\
\mathbf{d}\end{array}$ & $\begin{array}{c}\mathbf{2 5} \boldsymbol{\mu g} / \mathbf{m} \\
\mathbf{I}\end{array}$ & $\begin{array}{c}\mathbf{5 0} \\
\boldsymbol{\mathbf { g }} / \mathbf{m} \\
\mathbf{l}\end{array}$ & $\begin{array}{c}\mathbf{7 5} \\
\boldsymbol{\mu g} / \mathbf{m} \\
\mathbf{l}\end{array}$ & $\begin{array}{c}\mathbf{1 0 0} \\
\boldsymbol{\mu g} / \mathbf{m} \\
\mathbf{l}\end{array}$ & $\begin{array}{c}\mathbf{I C}_{\mathbf{5 0}} \boldsymbol{\mu M} \mathbf{m} \\
\mathbf{l}\end{array}$ \\
\hline 1. & 28.18 & 50.67 & 75.98 & 91.89 & 0.17 \\
\hline 2. & 28.19 & 51.67 & 67.90 & 77.87 & 0.21 \\
\hline 3. & 29.51 & 44.76 & 77.90 & 87.14 & 0.20 \\
\hline 4. & 41.01 & 50.00 & 56.19 & 64.28 & 0.18 \\
\hline 5. & 39.18 & 45.35 & 64.89 & 77.89 & 0.22 \\
\hline 6. & 45.02 & 47.61 & 56.66 & 68.09 & 0.18 \\
\hline 7. & 44.88 & 47.14 & 52.85 & 59.52 & 0.30 \\
\hline 8. & 42.65 & 47.14 & 51.90 & 89.52 & 0.19 \\
\hline 9. & 36.96 & 41.90 & 46.19 & 50.95 & 0.36 \\
\hline 10. & 41.23 & 45.23 & 50.47 & 54.76 & 0.29 \\
\hline 11. & 39.81 & 42.85 & 64.28 & 77.61 & 0.19 \\
\hline 12. & 39.33 & 43.33 & 51.90 & 68.09 & 0.23 \\
\hline 13. & 37.91 & 42.38 & 57.61 & 71.42 & 0.21 \\
\hline 14. & 37.44 & 41.42 & 52.85 & 66.19 & 0.24 \\
\hline $\begin{array}{c}\text { Ascorbic } \\
\text { acid* }\end{array}$ & 29.08 & 51.78 & 78.33 & 93.78 & 0.27 \\
\hline
\end{tabular}


Indo Global Journal of Pharmaceutical Sciences, 2017; 7(2): 126-133

Molecular docking studies: Molecular docking studies were analysed by AutoDock version 4.2.5.1 along with AutoDockTool (ADT). The Lamarckian Genetic Algorithm (LGA), considered one of the best docking method in the AutoDockTool was used which is capable of predicting binding orientation, position and conformations of the ligand [18]. The AutoGrid alogorithm was used to create 3D grid boxes which explore the chemistry of macromolecules in terms of binding energy [19]. Ligand PDB were prepared by Chem3D Ultra. AutoGrid is used to calculate detailed study of ligand in the actual docking target site [20]. Conformations of docked compounds were aligned in terms of hydrogen bonding, energy and hydrophobic interaction between ligand and receptor protein. Free binding energy of all synthesized compounds was calculated by docking scores [21]. For display of the receptor with ligand binding site and interaction of compounds with amino acids residues in the active sites PyMol software was used [20]. Acarbose in the crystal structure was docked as reference. Binding profile of docking is shown in Table no 6. Compound 1 shows maximum binding energy $(-8.30 \mathrm{kcal} / \mathrm{mol})$.

Table 6: Molecular docking results of target compounds (1-14)

\begin{tabular}{|c|c|c|}
\hline Compound no. & $\begin{array}{c}\text { Binding energy } \\
(\mathbf{k c a l} / \mathbf{m o l})\end{array}$ & $\begin{array}{c}\text { Inhibition } \\
\text { constant }(\boldsymbol{\mu M})\end{array}$ \\
\hline 1. & -8.03 & 1.29 \\
\hline 2. & -6.81 & 10.15 \\
\hline 3. & -5.94 & 44.30 \\
\hline 4. & -6.43 & 19.28 \\
\hline 5. & -6.52 & 16.67 \\
\hline 6. & -6.71 & 12.14 \\
\hline 7. & -6.28 & 24.99 \\
\hline 8. & -6.54 & 15.97 \\
\hline 9. & -7.44 & 3.50 \\
\hline 10. & -5.86 & 50.82 \\
\hline 11. & -6.53 & 16.21 \\
\hline 12. & -6.14 & 31.41 \\
\hline 13. & -6.52 & 16.59 \\
\hline 14. & -6.88 & 9.09 \\
\hline Acarbose & $\mathbf{- 1 0 . 8 2}$ & $\mathbf{0 . 0 1 1 7 2}$ \\
\hline
\end{tabular}

For docking analysis: The molecular studies was developed by AutoDockTools (ADT) (Sanner, 1999) version 1.5.6 and Autodock version 4.2.5.1programs; (Autodock, Autogrid, Copyright-1989-2012) from the Scripps Research Institute http://www.scripps.edu/mb/olson/doc/autodock. Ligand Compounds 3D structures were drawn using Chemsketch. For input into docking, the structure was saved as pdb file format; ligand bond torsion was aligned and saved in PDBQT file format. Three dimensional structures of Acarbose (PDB id: 1RP8), was obtained from the Protein Data Bank (PDB) http://www.pdb.org. Further, using ADT to remove crystal water, added Kollman and Gagteiger charges to each atom, and merged the non-polar hydrogen atoms to the protein structure. The distance between donor and acceptor atoms that form a hydrogen bond was defined as $1.9 \AA$ with a tolerance of $0.5 \AA$, and the acceptor-hydrogen-donor angle was not less than 120. The structures must be saved in PDBQT file format for accurately input into docking. [20].
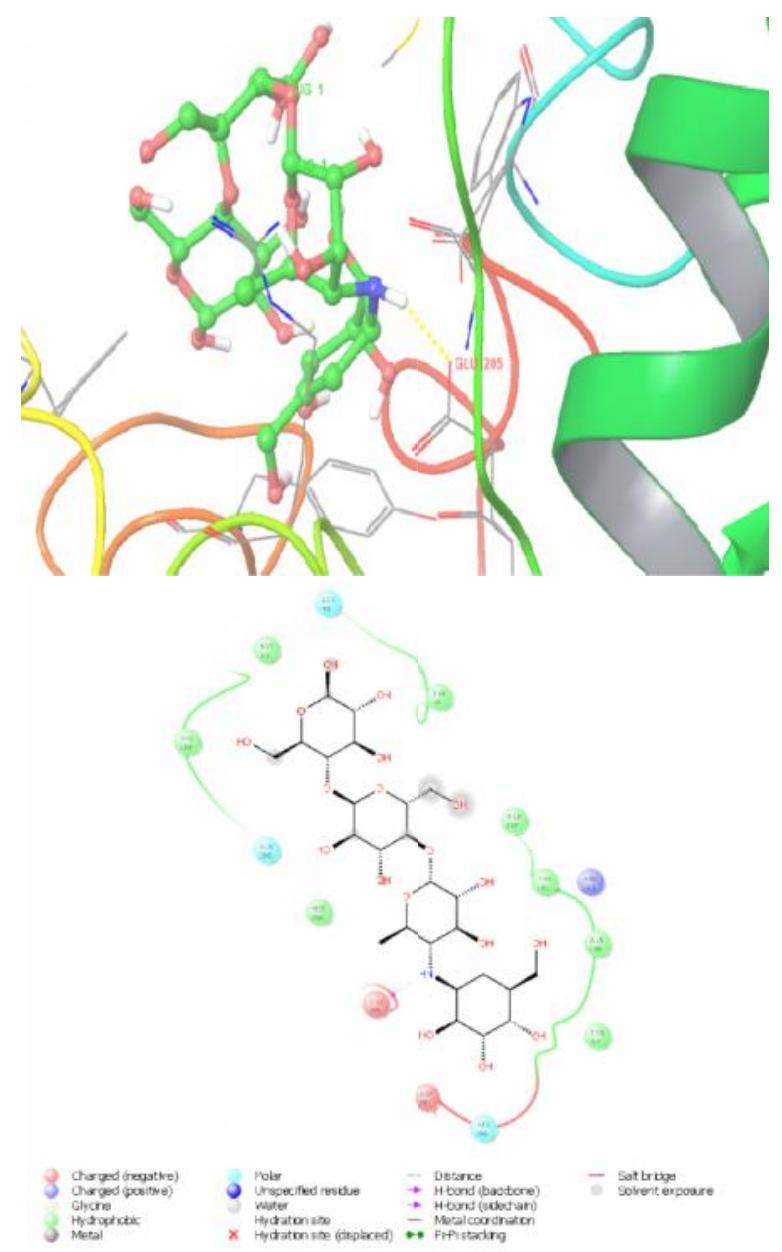

Fig 1: Binding residues of Acarbose

\section{Spectral data}

Intermediate (E)-3-(4-hydroxyphenyl) acryloyl chloride IR ( $\mathrm{KBr}$ pellets) $\mathrm{cm}^{-1}: 3033(\mathrm{C}-\mathrm{H}$ str., phenyl nucleus), 1598(C=C skeletal str., phenyl),3376(O-H str., phenol), 1631(C=C str., alkene). ${ }^{1} \mathbf{H}$ NMR (DMSO-d $\left.{ }_{6}, \boldsymbol{\delta p p m}\right)$ : 6.31-7.14 (q, 4H, Ar-H), 6.78 (d, 2H, CH=CH), $7.95(\mathrm{~s}, 1 \mathrm{H}$, Ar-CH).

Compound 1(E)-quinolin-8-yl 3-(4-hydroxyphenyl) acrylate IR ( $\mathrm{KBr}$ pellets) $\mathrm{cm}^{-1}: 3417$ (O-H str., phenol), 3034 (C-H str., aromatic ring), $1598(\mathrm{C}=\mathrm{C}$ str., skeletal vibration of phenyl nucleus), 1733 (C=Ostr., ester), 1632 ( $\mathrm{C}=\mathrm{C}$ str., alkene), 1309 (ring str., quinoline), 760 (C-H out of plane bending, quinoline), 1505 (C=N str., quinoline). ${ }^{1}$ H NMR (DMSO-d ${ }_{6}$, 
Indo Global Journal of Pharmaceutical Sciences, 2017; 7(2): 126-133

סppm): 6.81-7.14 (q, 4H, Ar-H), 6.81-8.89 (m, 6H, quinoline), $7.67(\mathrm{~d}, 2 \mathrm{H}, \mathrm{CH}=\mathrm{CH})$.

Compound 2 (E)-N-(4-flourophenyl)-3-(4-hydroxyphenyl) acrylamide

IR ( $\mathrm{KBr}$ pellets) $\mathrm{cm}^{-1}: 3748$ (O-H str., phenol), 3035 (C-H str., aromatic ring), $1599(\mathrm{C}=\mathrm{C}$ str., skeletal vibration of phenyl nucleus), 1632 ( $\mathrm{C}=\mathrm{O}$ str., $2^{\circ}$ amide), 1736 ( $\mathrm{C}=\mathrm{C}$ str., alkene), 3367 (N-H str., $2^{\circ}$ amide), 1015 (C-F str., aromatic), 1506 (N$\mathrm{H}$ bend). ${ }^{1} \mathbf{H}$ NMR (DMSO-d $\left.{ }_{6}, \mathbf{\delta p p m}\right):$ 6.64-7.64 (m, 8H, Ar$\mathrm{H}), 6.55(\mathrm{~d}, 2 \mathrm{H}, \mathrm{CH}=\mathrm{CH}), 7.08(\mathrm{~s}, 1 \mathrm{H}, \mathrm{Ar}-\mathrm{CH}), 7.94(\mathrm{~s}, 1 \mathrm{H}$, $\mathrm{NH})$.

\section{Compound 3 (E)-3-(4-hydroxyphenyl)-N-phenyl} acrylamide

IR (KBr pellets) $\mathrm{cm}^{-1}: 3748$ (O-H str., phenol), 3035(C-H str., aromatic ring), $1598(\mathrm{C}=\mathrm{C}$ str., skeletal vibration of phenyl nucleus), 1633 ( $\mathrm{C}=\mathrm{O}$ str., $2^{\circ}$ amide), 1739 ( $\mathrm{C}=\mathrm{C}$ str., alkene), 3365 (N-H str., $2^{\circ}$ amide), 1506 ( $\mathrm{N}-\mathrm{H}$ bend). ${ }^{1} \mathbf{H}$ NMR (DMSO-d $\left.{ }_{6}, \mathbf{\delta p p m}\right):$ 6.64-7.64 (m, 10H, Ar-H), 6.83 (d, 2H, $\mathrm{CH}=\mathrm{CH}), 7.51(\mathrm{~s}, 1 \mathrm{H}, \mathrm{Ar}-\mathrm{CH}), 7.96(\mathrm{~s}, 1 \mathrm{H}, \mathrm{NH})$.

Compound 4 (E)-N-(2-chlorophenyl)-3-(4-hydroxyphenyl) acrylamide

IR (KBr pellets) $\mathrm{cm}^{-1}: 3034$ (C-H str., aromatic ring), 1598 ( $\mathrm{C}=\mathrm{C}$ str., skeletal vibration of phenyl nucleus), $1631(\mathrm{C}=\mathrm{O}$ str., $2^{\circ}$ amide), $1733\left(\mathrm{C}=\mathrm{C}\right.$ str., alkene), 3367(N-H str., $2^{\circ}$ amide), 754 (C-Cl str., aromatic), 1505 (N-H bend). ${ }^{1} \mathbf{H}$ NMR (DMSO-d $\left.{ }_{6}, \mathbf{\delta p p m}\right):$ 6.64-7.60 (m, 8H, Ar-H), $6.84(\mathrm{~d}, 2 \mathrm{H}$, $\mathrm{CH}=\mathrm{CH}), 7.60(\mathrm{~s}, 1 \mathrm{H}, \mathrm{Ar}-\mathrm{CH}), 7.96(\mathrm{~s}, 1 \mathrm{H}, \mathrm{NH})$.

Compound 5 (E)-3-(4-hydroxyphenyl)-N-(2-nitrophenyl) acrylamide

IR ( $\mathrm{KBr}$ pellets) $\mathrm{cm}^{-1}: 3067$ (C-H str., aromatic ring), 1733 ( $\mathrm{C}=\mathrm{C}$ str., alkene), 1598 ( $\mathrm{C}=\mathrm{C}$ str., skeletal vibration of phenyl nucleus), 1630 ( $\mathrm{C}=\mathrm{O}$ str., $2^{\circ}$ amide), 3365 (N-H str., $2^{\circ}$ amide), 1362 $\left(\mathrm{NO}_{2}\right.$ sym. str., Ar- $\left.\mathrm{NO}_{2}\right), 1505$ (N-H bend). ${ }^{1} \mathbf{H}$ NMR (DMSO-d ${ }_{6}$, oppm): 6.68-7.97 (m, 8H, Ar-H), 6.84 (d, $2 \mathrm{H}, \mathrm{CH}=\mathrm{CH}), 7.59(\mathrm{~s}, 1 \mathrm{H}, \mathrm{Ar}-\mathrm{CH}), 7.97(\mathrm{~s}, 1 \mathrm{H}, \mathrm{NH})$.

Compound 6 (E)-3-(4-hydroxyphenyl)-N-(3-nitrophenyl) acrylamide

IR ( $\mathrm{KBr}$ pellets) $\mathrm{cm}^{-1}: 3067$ (C-H str., aromatic ring), 1733 ( $\mathrm{C}=\mathrm{C}$ str., alkene), 1598 ( $\mathrm{C}=\mathrm{C}$ str., skeletal vibration of phenyl nucleus), 1631 (C=O str., $2^{\circ}$ amide), 1310 (C-N str., aromatic), 3370 (N-H str., $2^{\circ}$ amide), $1356\left(\mathrm{NO}_{2}\right.$ sym. str., $\left.\mathrm{Ar}-\mathrm{NO}_{2}\right), 1505$ (N-H bend). ${ }^{1} \mathbf{H}$ NMR (DMSO-d $\left.{ }_{6}, \boldsymbol{\delta p p m}\right):$ 6.67-8.01 (m, 8H, $\mathrm{Ar}-\mathrm{H}), 6.67$ (d, 2H, CH=CH), 7.56 (s,1H, Ar-CH), 8.01 (s, 1H, $\mathrm{NH})$.
Compound 7 (E)-N-(2, 4-dinitrophenyl)-3-(4hydroxyphenyl) acrylamide

IR ( $\mathrm{KBr}$ pellets) $\mathrm{cm}^{-1}: 3107$ (C-H str., aromatic ring), 1733 ( $\mathrm{C}=\mathrm{C}$ str., alkene), 1599 ( $\mathrm{C}=\mathrm{C}$ str., skeletal vibration of phenyl nucleus), 1630 ( $\mathrm{C}=\mathrm{O}$ str., $2^{\circ}$ amide),3336 (N-H str., $2^{\circ}$ amide), 1333( $\mathrm{NO}_{2}$ sym. str., Ar- $\left.\mathrm{NO}_{2}\right), 1501$ (N-H bend). ${ }^{1} \mathbf{H}$ NMR (DMSO-d ${ }_{6}$, oppm): 7.12-8.80 (m, 7H, Ar-H), 7.24 (s,1H, Ar$\mathrm{CH}), 8.14$ (s, 1H, NH).

Compound 8 (E)-N-(4-chlorophenyl)-3-(4-hydroxyphenyl) acrylamide

IR ( $\mathrm{KBr}$ pellets) $\mathrm{cm}^{-1}: 3034$ (C-H str., aromatic ring), 1734 ( $\mathrm{C}=\mathrm{C}$ str., alkene), 1598 ( $\mathrm{C}=\mathrm{C}$ str., skeletal vibration of phenyl nucleus), 1631 ( $\mathrm{C}=\mathrm{O}$ str., $2^{\circ}$ amide), 3368 (N-H str., $2^{\circ}$ amide), 1505 (N-H bend). ${ }^{1} \mathbf{H}$ NMR (DMSO-d 6 , $\left.\mathbf{~} \mathbf{p p m}\right)$ : 6.65$7.66(\mathrm{~m}, 8 \mathrm{H}, \mathrm{Ar}-\mathrm{H}), 6.86(\mathrm{~d}, 2 \mathrm{H}, \mathrm{CH}=\mathrm{CH}), 7.51(\mathrm{~s}, 1 \mathrm{H}, \mathrm{Ar}-$ $\mathrm{CH}), 7.94$ (s, 1H, NH).

Compound 9 (E)-N-(2,6-dimethylphenyl)-3-(4hydroxyphenyl) acrylamide

IR ( $\mathrm{KBr}$ pellets) $\mathrm{cm}^{-1}: 3034$ (C-H str., aromatic ring), 1734 ( $\mathrm{C}=\mathrm{C}$ str., alkene), 1598 ( $\mathrm{C}=\mathrm{C}$ str., skeletal vibration of phenyl nucleus), 1631 ( $\mathrm{C}=\mathrm{O}$ str., $2^{\circ}$ amide), 3365 (N-H str., $2^{\circ}$ amide), 2831 ( $\left.\mathrm{Ar}^{-} \mathrm{CH}_{3}\right), 1505$ (N-H bend). ${ }^{1} \mathrm{H}$ NMR (DMSO$\left.\mathbf{d}_{\mathbf{6}}, \mathbf{\delta p p m}\right):$ 6. 65-7.13 (m, 7H, Ar-H), $6.83(\mathrm{~d}, 2 \mathrm{H}, \mathrm{CH}=\mathrm{CH})$, $7.51(\mathrm{~s}, 1 \mathrm{H}, \mathrm{Ar}-\mathrm{CH}), 7.93(\mathrm{~s}, 1 \mathrm{H}, \mathrm{NH}), 2.41\left(\mathrm{~m}, 6 \mathrm{H}, \mathrm{Ar}-\mathrm{CH}_{3}\right)$.

Compound 10 (E)-N-(2-flourophenyl)-3-(4-hydroxyphenyl) acrylamide

IR ( $\mathrm{KBr}$ pellets) $\mathrm{cm}^{-1}: 3034$ (C-H str., aromatic ring), 1733 ( $\mathrm{C}=\mathrm{C}$ str., alkene), 1598 ( $\mathrm{C}=\mathrm{C}$ str., skeletal vibration of phenyl nucleus), 1631 ( $\mathrm{C}=\mathrm{O}$ str., $2^{\circ}$ amide), 3368 (N-H str., $2^{\circ}$ amide), 1015 (C-F str., aromatic), 1505 (N-H bend). ${ }^{1} \mathbf{H}$ NMR (DMSO-d $\left.{ }_{6}, \mathbf{\delta p p m}\right):$ 6.61-7.63 (m, 8H, Ar-H), 6.83 (d, 2H, $\mathrm{CH}=\mathrm{CH}), 7.52(\mathrm{~s}, 1 \mathrm{H}, \mathrm{Ar}-\mathrm{CH}), 7.95(\mathrm{~s}, 1 \mathrm{H}, \mathrm{NH})$.

Compound $11 \quad$ (E)-3-(4-hydroxyphenyl)-N-(2methoxyphenyl) acrylamide

IR ( $\mathrm{KBr}$ pellets) $\mathrm{cm}^{-1}: 3035$ (C-H str., aromatic ring), 1733 ( $\mathrm{C}=\mathrm{C}$ str., alkene), 1598 ( $\mathrm{C}=\mathrm{C}$ str., skeletal vibration of phenyl nucleus), $1630\left(\mathrm{C}=\mathrm{O}\right.$ str., $2^{\circ}$ amide), 3415 (N-H str., $2^{\circ}$ amide), 1280 (C-O-C str., Ar-OCH ${ }_{3}$ ), 1505 (N-H bend). ${ }^{1} \mathrm{H}$ NMR (DMSO-d $\left.{ }_{6}, \mathbf{\delta p p m}\right):$ 6.79-7.52 (m, 8H, Ar-H), 6.84 (d, $2 \mathrm{H}, \mathrm{CH}=\mathrm{CH}), 7.52(\mathrm{~s}, 1 \mathrm{H}, \mathrm{Ar}-\mathrm{CH}), 7.95(\mathrm{~s}, 1 \mathrm{H}, \mathrm{NH}), 3.75$ (t, $\left.3 \mathrm{H}, \mathrm{OCH}_{3}\right)$.

Compound $12 \quad$ (E)-3-(4-hydroxyphenyl)-N-(3methoxyphenyl) acrylamide

IR ( $\mathrm{KBr}$ pellets) $\mathrm{cm}^{-1}: 3067$ (C-H str., aromatic ring), 1733 ( $\mathrm{C}=\mathrm{C}$ str., alkene), 1598 ( $\mathrm{C}=\mathrm{C}$ str., skeletal vibration of phenyl nucleus), 1631 ( $\mathrm{C}=\mathrm{O}$ str., $2^{\circ}$ amide), 3371 (N-H str., $2^{\circ}$ 
Indo Global Journal of Pharmaceutical Sciences, 2017; 7(2): 126-133

amide), 1280 (C-O-C str., $\mathrm{Ar}-\mathrm{OCH}_{3}$ ), 1505 (N-H bend). ${ }^{1} \mathbf{H}$

NMR (DMSO-d 6 , סppm): 6.65-7.51 (m, 8H, Ar-H), 6.84 (d, $2 \mathrm{H}, \mathrm{CH}=\mathrm{CH}), 7.51(\mathrm{~s}, 1 \mathrm{H}, \mathrm{Ar}-\mathrm{CH}), 7.97(\mathrm{~s}, 1 \mathrm{H}, \mathrm{NH}), 3.73(\mathrm{t}$, $3 \mathrm{H}, \mathrm{OCH}_{3}$ ).

\section{Compound $13 \quad$ (E)-3-(4-hydroxyphenyl)-N-(4-} methoxyphenyl) acrylamide

IR ( $\mathrm{KBr}$ pellets) $\mathrm{cm}^{-1}: 3035$ (C-H str., aromatic ring), 1733 ( $\mathrm{C}=\mathrm{C}$ str., alkene), $1598(\mathrm{C}=\mathrm{C}$ str., skeletal vibration of phenyl nucleus), 1631 ( $\mathrm{C}=\mathrm{O}$ str., $2^{\circ}$ amide), 3362 (N-H str., $2^{\circ}$ amide), 1280 (C-O-C str., $\left.\mathrm{Ar}-\mathrm{OCH}_{3}\right), 1507$ (N-H bend). ${ }^{1} \mathbf{H}$ NMR (DMSO-d 6 , סppm): 6.67-7.56 (m, 8H, Ar-H), 6.83 (d, $2 \mathrm{H}, \mathrm{CH}=\mathrm{CH}), 7.56$ (s,1H, Ar-CH), 7.94 (s, 1H, NH), 3.75 (t, $3 \mathrm{H}, \mathrm{OCH}_{3}$ ).

\section{Compound $14 \quad$ (E)-N-(2,4-dimethylphenyl)-3-(4-} hydroxyphenyl) acrylamide

IR ( $\mathrm{KBr}$ pellets) $\mathrm{cm}^{-1}: 3068$ (C-H str., aromatic ring), 1744 ( $\mathrm{C}=\mathrm{C}$ str., alkene), 1598 ( $\mathrm{C}=\mathrm{C}$ str., skeletal vibration of phenyl nucleus), 1630 ( $\mathrm{C}=\mathrm{O}$ str., $2^{\circ}$ amide), 3416 (N-H str., $2^{\circ}$ amide), 2830 ( $\left.\mathrm{Ar}-\mathrm{CH}_{3}\right), 1507$ (N-H bend). ${ }^{1} \mathbf{H}$ NMR (DMSOd 6 , סppm): 6.65-7.41 (m, 7H, Ar-H), $6.85(\mathrm{~d}, 2 \mathrm{H}, \mathrm{CH}=\mathrm{CH})$, 7.51 (s,1H, Ar-CH), 7.96 (s, 1H, NH), 2.36 (m, 6H, $\left.\mathrm{CH}_{3}-\mathrm{Ar}\right)$.

\section{CONCLUSION}

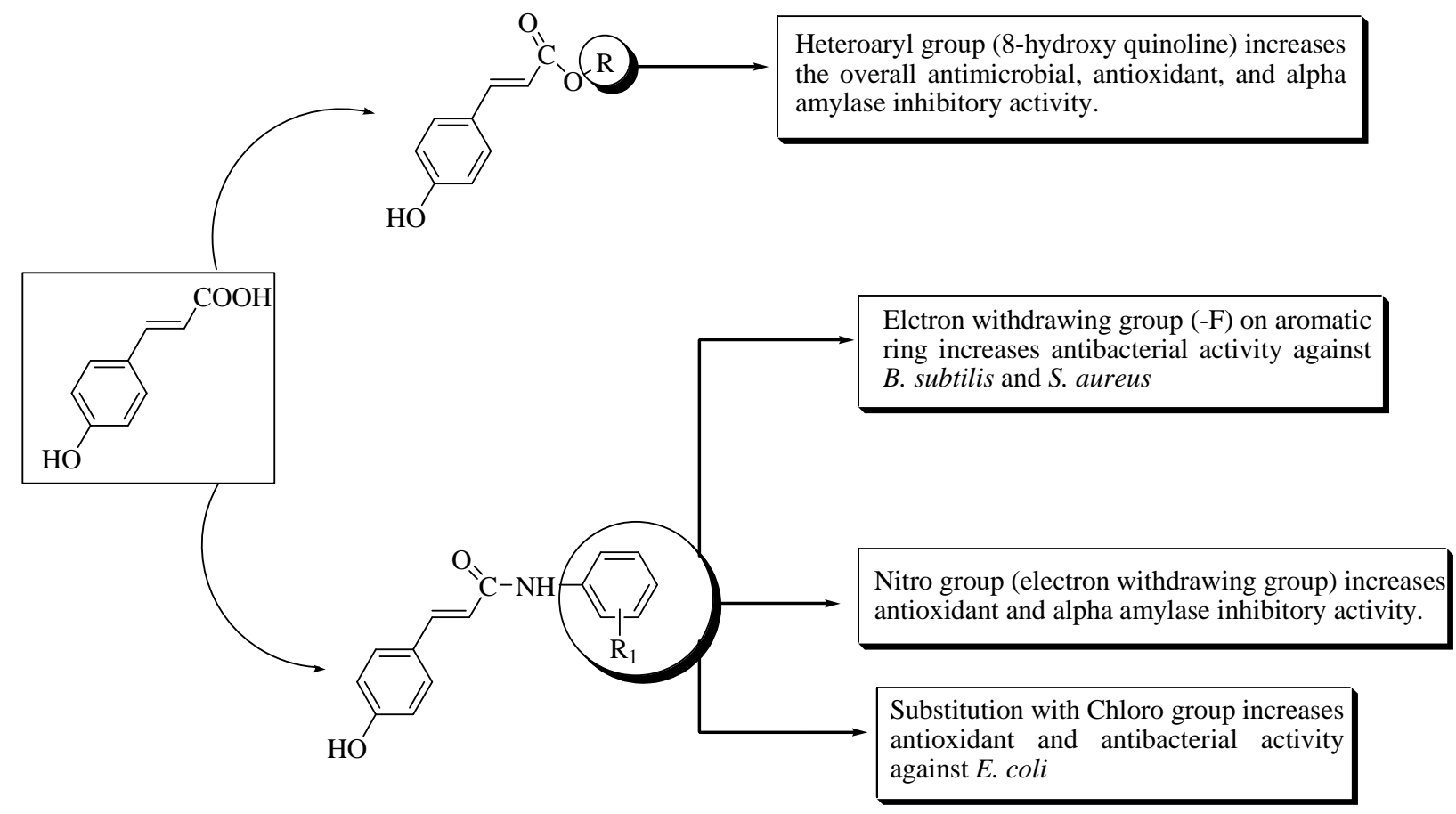

Fig 2: Structure-activity relationship of the $p$-coumaric acid derivatives. 


\section{Indo Global Journal of Pharmaceutical Sciences, 2017; 7(2): 126-133}

\section{REFERENCES}

1. C. Hano, S. Renouard, R. Molinie, C. Corbin, E. Barakzoy, J. Doussot, F. Lamblin, E.Laine. Flaxseed (Linum usitatissimum L.) extract as well as (+)-secoisolariciresinol diglucoside and its mammalian derivatives are potent inhibitors of $\alpha$-amylase activity. Bioorg. Med. Chem. Lett., 2013, 23: 3007-3012.

2. V. S.Patil, K. P. Nandre, S. Ghosh, V.J. Rao, B.A. Chopade, S.V. Bhosale, S.V. Bhosale. Synthesis and glycosidase inhibitory activity of novel (2-phenyl-4H-benzopyrimedo [2, 1-b]-thiazol-4yliden) acetonitrile derivatives. Bioorg. Med. Chem. Lett., 2012, 22: 7011-7014.

3. C. T. Kumarappan, T. N. Rao, S. C. Mandal. Polyphenolic extract of Ichnocarpus frutescens modifies hyperlipidemia status in diabetic rats. J. Cell Mol. Biol., 2007, 6(22), 175-187.

4. I. E. Alvarado, D. Navarro, E. Record, M. Asther, M. Asther, L.L. Meessen. Fungal biotransformation of p-coumaric acid into caffeic acid by Pycnoporus cinnabarinus: an alternative for producing a strong natural antioxidant. World J. Microbiol. Biotechnol., 2003, 19: $157-160$.

5. M. O. P. Bicudo, R. H. Ribani, T. Beta. Anthocyanins, phenolic acids and antioxidant properties of juçara fruits (Euterpe edulis M.) along the on-tree ripening process. Plant foods for human nutrition, 2014, 69(2), 142-147.

6. A. Khatkar, A. Nanda, P. Kumar, B. Narasimhan. Synthesis, antimicrobial evaluation and QSAR studies of p-coumaric acid derivatives.Arabian J. Chem., 2014: 1-12.

7. Y. Yao, X. Z. Cheng, L. X. Wang, S.H. Wang, G. Ren. Major phenolic compounds, antioxidant capacity and antidiabetic potential of rice bean (Vigna umbellata L.) in China. Int. J.Mol. Sci., 2012, 13: 2707-2716

8. M. Deepa, T. Sureshkumar, P. K. Satheeshkumar, S. Priya. Antioxidant rich Morus alba leaf extract induces apoptosis in human colon and breast cancer cells by the downregulation of nitric oxide produced by inducible nitric oxide synthase. Nutrition and cancer, 2013,65: 305-310.

9. S. J. Pragasam, M. Rasool. Dietary component p-coumaric acid suppresses monosodium urate crystal-induced inflammation in rats.Inflammation Res., 2013, 62: 489-498.

10. L. Liu, B. Lu, D. Xia, Y. Zhang. Evaluation of antihypertensive and antihyperlipidemic effects of bamboo shoot angiotensin converting enzyme inhibitory peptide in vivo. J. Agri. Food Chem., 2012, 60: 11351-11358.

11. M. P. De Barros, M. Lemos, E. L. Maistro, M. F. Leite, J. P. B. Sousa, J. K. Bastos, S. F. De Andrade. Evaluation of antiulcer activity of the main phenolic acids found in Brazilian Green Propolis. J. Ethnopharmacol., 2008, 120: 372-377.

12. K. Song, S. M. An, M. Kim, J. S. Koh, Y. C. Boo. Comparison of the antimelanogenic effects of p-coumaric acid and its methyl ester and their skin permeabilities. J. Dermatol. Sci., 2011, 63: 17-22.

13. B. Nickavar, G. Amin. Bioassay-guided separation of a $\alpha-$ amylase inhibitor anthocyanin from Vaccinium arctostaphylos berries. Zeitschrift für Naturforschung C, 2010, 65: 567-570.

14. A. Duraiswamy, D.Shanmugasundaram, C. S. Sasikumar, S. M. Cherian, K. M. Cherian. Development of an antidiabetic formulation (ADJ6) and its inhibitory activity against $\alpha$-amylase and $\alpha$ glucosidase. J. Tradition. Complement. Med., 2015: 1-5.

15. P. Kumar, M.Mehta, S. Satija, M. Garg. Enzymatic in vitro Anti-diabetic Activity of Few Traditional Indian Medicinal Plants. J. Biol. Sci., 2013, 13: 540-544.

16. N. Malik, P. Dhiman, P. K. Verma, A. Khatkar. Design, synthesis, and biological evaluation of thiourea and guanidine derivatives of pyrimidine-6-carboxylate. Res. Chem. Intermed., 2014, 7: $1-13$.

17. P. Dhiman, N. Malik, P. K. Verma, A. Khatkar. Synthesis and biological evaluation of thiazolo and imidazo N-(4-nitrophenyl)-7methyl-5-aryl-pyrimidine-6 carboxamide derivatives. Res. Chem. Intermed., 2015, 8: 1-13.

18. G. L. Chen, L. H. Wang, J. Wang, K. Chen, M. Zhao, Z. Z. Sun, S. Wang, H. L Zheng, J. Y. Yang, C. F. Wu. Discovery of a small molecular compound simultaneously targeting RXR and HADC: Design, synthesis, molecular docking and bioassay. Bioorg. Med. Chem. Lett., 2013, 23: 3891-3895.

19. P. Yadav, P. Singh, A. K. Tewari. Design, synthesis, docking and anti-inflammatory evaluation of novel series of benzofuran based prodrugs. Bioorg. Med. Chem. Lett., 2014, 24: 2251-2255.

20. D. Kathirvelan, J. Haribabu, B. S. R. Reddy, C. Balachandran, V. Duraipandiyan. Facile and diastereoselective synthesis of 3, 2'spiropyrrolidine-oxindoles derivatives, their molecular docking and antiproliferative activities. Bioorg. Med. Chem. Lett., 2015, 25: 389399.

21. S. S. Sulthana, S. A. Antony, C. Balachandran, S. S. Shafi. Thiophene and benzodioxole appended thiazolyl-pyrazoline compounds: Microwave assisted synthesis, antimicrobial and molecular docking studies.Bioorg. Med. Chem. Lett., 2015, 25: 2753 2757.

Indo Global Journal of Pharmaceutical Sciences( ISSN 22491023 ; UGC Journal No.: 44477; CODEN- IGJPAI; NLM ID: 101610675) indexed and abstracted in EMBASE(Elsevier), UGC Journal List, National Library of Medicine (NLM) Catalog, Elsevier( EMBASE), ResearchGate, Publons, CAS (ACS), Index Copernicus, Google Scholar and many more. For further details, visit http://iglobaljournal.com 